\title{
Entropy Generation In The Viscous Layer Of A Turbulent Channel Flow
}

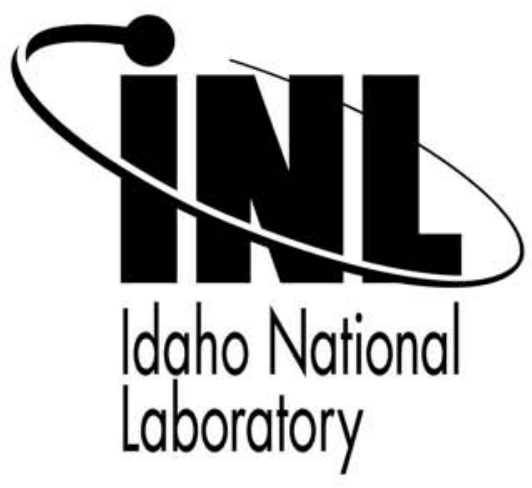

Donald M. McEligot Edmond J. Walsh Eckart Laurien

September 2006
This is a preprint of a paper intended for publication in a journal or proceedings. Since changes may not be made before publication, this preprint should not be cited or reproduced without permission of the author. This document was prepared as an account of work sponsored by an agency of the United States Government. Neither the United States Government nor any agency thereof, or any of their employees, makes any warranty, expressed or implied, or assumes any legal liability or responsibility for any third party's use, or the results of such use, of any information, apparatus, product or process disclosed in this report, or represents that its use by such third party would not infringe privately owned rights. The views expressed in this paper are not necessarily those of the United States Government or the sponsoring agency. 


\title{
Entropy generation in the viscous layer of a turbulent channel flow
}

\author{
Donald M. McEligot ${ }^{1,2,3}$, Edmond J. Walsh ${ }^{4}$ and Eckart Laurien ${ }^{2}$ \\ ${ }^{1}$ Aero. Mech. Engr. Dept., Univ. Arizona, Tucson, Ariz. 85721 USA \\ ${ }^{2}$ Inst. für Kernenergetik u. Energiesysteme (IKE), Uni. Stuttgart, D-70550 Stuttgart, Germany \\ ${ }^{3}$ Idaho National Laboratory, Idaho Falls, Ida. 83415-3885 USA \\ ${ }^{4}$ Mech. Aero. Engr. Dept., Univ. Limerick, Limerick, Ireland
}

\begin{abstract}
The local (pointwise) entropy generation rate per unit volume $\mathrm{S}$ "' is a key to improving many energy processes and applications. Entropy generation due to friction occurs from viscous dissipation of mean-flow kinetic energy (called "direct dissipation") and dissipation of turbulent kinetic energy into thermal energy ("indirect" or turbulent dissipation). The objective of the present study is to compare two approaches for the prediction of S"' for the viscous layer in near asymptotic (high Reynolds number) turbulent flows. By employing available direct numerical simulations (DNS) it was found that about two-thirds of the entropy generation occurs in this layer. A popular approximate approach does not agree with the result from the more exact evaluation of $\mathrm{S}$ "' but its integral falls within about four per cent at the edge of the viscous layer.
\end{abstract}

\section{Introduction}

The local (pointwise) entropy generation rate per unit volume $\mathrm{S}$ "' is a key to improving many energy processes and applications [Bejan, 1982]. In developing his reciprocal relations for irreversible processes, Onsager [1931] extended Lord Rayleigh's "principle of least dissipation of energy" and indicated that the rate of increase of entropy plays the role of a potential. Thus, entropy generation (or "production" [Prigogine, 1978]) may be used as a parameter to measure a system's departure from reversibility. Bejan has suggested that real systems which owe their thermodynamic imperfections to fluid flow, heat transfer and mass transfer irreversibilities be optimized by minimizing their entropy generation. This approach has been applied to compact heat exchangers, power plants, natural convection, rotating bodies, enhanced heat transfer surfaces, impinging jets, convection in general and other thermal systems.

Kock and Herwig [2005] suggest that predicting the efficient use of energy in thermal systems requires accounting for the second law of thermodynamics since the loss of available work [Kestin, 1980] is proportional to the amount of entropy produced (e.g., via the Gouy [1889]-Stodola [1910] theorem cited by Bejan). Therefore, apparatus producing less entropy by irreversibilities destroys less available work, increasing the efficiency. Neumann, von Wolfersdorf and Weigand [2005], Kock and Herwig and others are using computational fluid dynamics (CFD) codes to predict entropy generation for optimization by minimizing it. Since $\mathrm{S}^{\prime \prime}$ determines the localized contribution to energy losses or reduction in the availability of energy [Clausius, 1887; Lichty, 1936], insight into the dominant loss sources and their locations can allow reducing them intelligently, thereby improving efficiency. These CFD studies seek to identify the regions of maximum entropy production so they may be attacked and reduced.

In the present study, we examine entropy generation due to shear stresses in idealized fully-developed turbulent channel flows between infinitely-wide flat plates. We concentrate on the viscous layer because it is typically the region where the largest gradients occur and the production of turbulence is greatest. Following Bradshaw [1975], we are here defining the 
viscous layer as the region where viscous effects are significant, but not necessarily dominant, typically to $\mathrm{y}^{+}$about 30 in a classical zero-pressure gradient case (it includes the "laminar" and buffer sublayers in some investigators's terminology). The quantity y is the normal distance from the wall and the superscript ${ }^{+}$here and later represents normalization by wall units, kinematic viscosity $v$ and friction velocity $u_{\tau}\left(=\left(\tau_{\mathrm{W}} / \rho\right)^{1 / 2}\right.$ with $\tau_{\mathrm{W}}$ symbolizing the mean wall shear stress and $\rho$ the fluid density). The major resistances to momentum, energy and mass transfer occur in this layer -- and the pointwise entropy generation rate is greatest here as well.

Key relations for evaluating entropy generation are presented in the Background section which follows. Proposals to estimate the pointwise contribution of entropy generation rates due to friction in incompressible turbulent wall flows include (1) evaluation of the fluctuating gradients forming the dissipation term in the turbulent enthalpy equation and (2) an approximate analogy to laminar flow employing assumed boundary layer (and other) approximations [Schlichting, eq. 23.8d, 1968]. Accordingly, the objective of the present study is to compare these two approaches for the viscous layer in near asymptotic (high Reynolds number) turbulent flows. The bases of the examination are the direct numerical simulations (DNS) by Abe, Kawamura and Matsuo [2001]. After comparison for the pointwise and integral results, we then summarize with concluding remarks.

\section{Background}

Entropy appears in the second law of thermodynamics which can be written for a flowing open system, in terms of the "rate of creation" of entropy [London, 1960], as

$$
\operatorname{RoC}(\mathrm{S})=\mathrm{S}_{\mathrm{out}}+\left(\mathrm{dS}_{\mathrm{cV}} / \mathrm{dt}\right)-\mathrm{S}_{\text {in }} \geq \sum(\dot{\mathrm{Q}} / \mathrm{T})
$$

where $\mathrm{S}_{\mathrm{in} / \text { out }}$ is the rate of entropy convection into or out of the system, $\mathrm{S}_{\mathrm{cv}}$ is the entropy

stored in the control volume, $\mathrm{t}$ is time, $\dot{\mathrm{Q}}$ is the rate of heat transfer into the control volume and $\mathrm{T}$ is the absolute temperature of the thermal reservoir from which this heat transfer comes. As a measure of the irreversibility, Bejan [1982] and others define an entropy generation rate or rate of production of entropy [Reynolds and Perkins, 1970]

$$
\mathrm{S}_{\text {gen }}=\sum(\dot{\mathrm{m}} \mathrm{s})_{\text {out }}+\left(\mathrm{dS}_{\mathrm{cv}} / \mathrm{dt}\right)-\sum(\dot{\mathrm{m}} \mathrm{s})_{\text {in }}-\sum(\dot{\mathrm{Q}} / \mathrm{T}) \geq 0
$$

which can be seen to be the inequality, if any, between $\operatorname{RoC}(\mathrm{S})$ and the reversible portion of entropy transfer with heat into the system. (The symbol $\dot{\mathrm{m}}$ represents the mass flow rate.) Possible irreversible processes are recognized to include friction, heat transfer with significant temperature gradients, combustion, etc.

For an isothermal, laminar pipe flow with no external heating imposed, Bejan [1982] and others suggest that the volumetric entropy generation rate $S^{\prime \prime}$ can be estimated by evaluating the viscous dissipation function $\Phi$ for the flow,

$$
\mathrm{S}^{\prime \prime \prime}\{\mathrm{y}\}=(\mu \Phi / \mathrm{T})=\mu(\partial \mathrm{U} / \partial \mathrm{y})^{2} / \mathrm{T}
$$


The symbol $\mu$ denotes absolute viscosity. Throughout the remainder of this paper, the streamwise velocity is represented as $U+u$, where upper and lower case letters symbolize its mean value and the fluctuation about it, respectively; the wall-normal velocity $\mathrm{V}+\mathrm{v}$ is treated in a similar fashion. (The braces \{\} are used to indicate that $\mathrm{S} " '$ is considered to be a function of y.)

The time-mean value of $\mu \Phi$ at a point in a flow with turbulent fluctuations may be expanded to $\mu \Phi+\rho \varepsilon$ where the former represents viscous dissipation of mean-flow kinetic energy (called "direct dissipation") and the latter represents dissipation of turbulent kinetic energy into thermal energy ("indirect" or turbulent dissipation) [Cebeci and Bradshaw, 1984; Gersten and Herwig, 1992; Schlichting and Gersten, 1997, 2000],

$$
\rho \varepsilon=2 \mu\left[\overline{\left(\frac{\partial \mathrm{u}}{\partial \mathrm{x}}\right)^{2}}+\overline{\left(\frac{\partial \mathrm{v}}{\partial \mathrm{y}}\right)^{2}}+\overline{\left(\frac{\partial \mathrm{w}}{\partial \mathrm{z}}\right)^{2}}\right]+\mu\left[\overline{\left(\frac{\partial \mathrm{u}}{\partial \mathrm{y}}+\frac{\partial \mathrm{v}}{\partial \mathrm{x}}\right)^{2}}+\overline{\left(\frac{\partial \mathrm{v}}{\partial \mathrm{z}}+\frac{\partial \mathrm{w}}{\partial \mathrm{y}}\right)^{2}}+\overline{\left(\frac{\partial \mathrm{w}}{\partial \mathrm{x}}+\frac{\partial \mathrm{u}}{\partial \mathrm{z}}\right)^{2}}\right]
$$

with $\mathrm{z}$ and $\mathrm{w}$ being the spanwise coordinate and the turbulent fluctuation in that direction, respectively. When expressed in wall units, the pointwise entropy generation rate for a fullydeveloped turbulent flow between infinitely-wide parallel plates can be written as

$$
\left(\mathrm{S}^{\prime \prime \prime}\left\{\mathrm{y}^{+}\right\}\right)^{+}=\left(\partial \mathrm{U}^{+} / \partial \mathrm{y}^{+}\right)^{2}+\varepsilon^{+}
$$

where $\left(\mathrm{S}^{\prime \prime \prime}\right)^{+}$is defined as $\mathrm{T} v \mathrm{~S}^{\prime \prime \prime} /\left(\rho \mathrm{u}_{\tau}{ }^{4}\right)$ and $\varepsilon^{+}$is $v \varepsilon / \mathrm{u}_{\tau}^{4}$ while $\mathrm{x}$ is the streamwise coordinate. The mean velocity components $\mathrm{V}$ and $\mathrm{W}$ are identically zero for this idealization.

The prediction of pointwise $\left(\mathrm{S}^{\prime \prime}\left\{\mathrm{y}^{+}\right\}\right)^{+}$is desired to identify regions where most losses occur (large values of $\mathrm{S}^{\prime \prime}$ ) and to deduce the entropy generation rate per unit surface area and, ultimately, $\mathrm{S}^{\prime}$ or S over the entire surface. In wall coordinates, the entropy generation per unit surface area can be evaluated as

$$
\left(\mathrm{S}^{\prime \prime}\left\{\mathrm{y}^{+}\right\}\right)^{+}=\left(\mathrm{TS}^{\prime \prime} /\left(\rho \mathrm{u}^{3}\right)\right)=\int_{0}^{\mathrm{y}^{+}}\left(\mathrm{S}^{\prime \prime \prime}\left\{\mathrm{y}^{+}\right\}\right)^{+} \mathrm{dy} \mathrm{y}^{+}
$$

Previous studies of turbulent flows with favorable streamwise pressure gradients have been summarized by Narasimha and Sreenivasan [1979], Spalart [1986], McEligot and Eckelmann [2006] and others. Based on definitions, continuity and momentum equations and empirical relations, one can form approximate relations between some of the non-dimensional parameters suggested as governing the flows. Streamwise acceleration in a boundary layer is often represented by an acceleration parameter [Kline et al., 1967] defined as

$$
K_{\mathrm{v}}=\left(v / \mathrm{U}_{\infty}^{2}\right) d \mathrm{U}_{\infty} / \mathrm{dx}
$$

The subscript infinity indicates evaluation in the freestream. For a boundary layer flow, one can show $\mathrm{K}_{\mathrm{v}}=-\left(\mathrm{c}_{\mathrm{f}} / 2\right)^{3 / 2} \mathrm{~K}_{\mathrm{p}}$ where $\mathrm{c}_{\mathrm{f}}$ is the skin friction coefficient, defined as $\left(2 \tau_{\mathrm{W}} /\left(\rho \mathrm{U}_{\infty}{ }^{2}\right)\right)$. For fully-developed flow in a duct, $\mathrm{K}_{\mathrm{v}}$ is zero by definition and the pressure-gradient parameter may be estimated as 


$$
\mathrm{K}_{\mathrm{p}}=\left(v / \rho \mathrm{u}_{\tau}^{3}\right) \mathrm{dp} / \mathrm{dx} \approx-20.1 \operatorname{Re}_{\mathrm{D}, \mathrm{h}}{ }^{-(7 / 8)}
$$

by employing a Blasius correlation [Patel, 1965]. The letter $\mathrm{p}$ denotes static pressure and the Reynolds number $\mathrm{Re}_{\mathrm{D}, \mathrm{h}}$ here is based on the bulk or mixed mean velocity and on the hydraulic diameter $\mathrm{D}_{\mathrm{h}}$ calculated as four times the cross sectional area divided by the "wetted" perimeter of the duct. Consequently, some authors may have interpreted "pressure-gradient effects" as "low-Reynolds-number effects" and others may not have realized that their fully-developed internal flow could have entailed significant streamwise pressure gradients. The distance $y_{c}$ to the centerplane, centerline or other thickness measure can be represented as

$$
\mathrm{y}_{\mathrm{c}}^{+}=\left(\mathrm{y}_{\mathrm{c}} \mathrm{u}_{\tau} / v\right)=\left(\mathrm{y}_{\mathrm{c}} / \mathrm{D}_{\mathrm{h}}\right) \operatorname{Re}_{\mathrm{D}, \mathrm{h}}\left(\mathrm{c}_{\mathrm{f}} / 2\right)^{1 / 2} \approx 0.20\left(\mathrm{y}_{\mathrm{c}} / \mathrm{D}_{\mathrm{h}}\right) \operatorname{Re}_{\mathrm{D}, \mathrm{h}}{ }^{7 / 8}
$$

This quantity is also denoted as $\operatorname{Re}_{\tau}\left(=\mathrm{y}_{\mathrm{c}} \mathrm{u}_{\tau} / \nu\right)$ by some investigators (e.g., Kim, Moin and Moser [1987]).

The likelihood of streamwise pressure gradients affecting the viscous layer was discussed by McEligot and Eckelmann [2006]. The governing momentum equation may be written

$$
\mathrm{U}^{+}\left(\partial \mathrm{U}^{+} / \partial \mathrm{x}^{+}\right)+\mathrm{V}^{+}\left(\partial \mathrm{U}^{+} / \partial \mathrm{y}^{+}\right)=-\mathrm{K}_{\mathrm{p}}+\left(\partial \tau^{+} / \partial \mathrm{y}^{+}\right)
$$

From the momentum equation, one sees the distribution of $\partial \tau^{+}\left\{y^{+}\right\} / \partial y^{+}$will be a function of $\mathrm{K}_{\mathrm{p}}$ alone if the convective terms are zero or negligible. Fully-developed flows in tubes, channels and parallel plate ducts inherently satisfy this requirement.

Near the wall, the solution for the total shear stress variation can be approximated [Julien, Kays and Moffat, 1969; Finnicum and Hanratty, 1988] as

$$
\tau^{+}\left\{\mathrm{y}^{+}\right\}=\left(\tau\left\{\mathrm{y}^{+}\right\} / \tau_{\mathrm{W}}\right)=1+\mathrm{K}_{\mathrm{py}}+\left[1-\left(\mathrm{cf} /\left(2 \mathrm{y}^{+}\right)\right) \int_{0}^{\mathrm{y}^{+}}\left(\mathrm{U}^{+}\right)^{2} \mathrm{dy}{ }^{+}\right]
$$

For a fully-developed flow in a duct or tube, the convective terms become zero as noted and this solution reduces to

$$
\tau^{+}\left\{\mathrm{y}^{+}\right\}=\left(\tau\left\{\mathrm{y}^{+}\right\} / \tau_{\mathrm{w}}\right)=1+\mathrm{K}_{\mathrm{p}} \mathrm{y}^{+}
$$

With $-\mathrm{K}_{\mathrm{p}} \approx 0$, the $\mathrm{x}$-momentum equation reduces to $\tau^{+}\left\{\mathrm{y}^{+}\right\} \approx 1$ near the wall, i.e., the constant shear layer assumption becomes valid, provided the flow thickness is "large" enough. For the effect of a pressure gradient to be negligible in the viscous layer, one could establish a criterion that $\tau^{+}$still be greater than 0.95 or such at its edge (say $y^{+} \approx 30$ ). This constraint translates to requirements such as $-K_{p}<0.0017, \operatorname{Re}_{\tau}>600$ and, for tubes or ducts, $\operatorname{ReD}, \mathrm{h}>$ 46,000. McEligot and Eckelmann suggest that, for the viscous layer behavior to be similar in various geometries and flows, one needs (1) the viscous layer to be small relative to geometric scales in the flow and (2) to have the same distribution of $\partial \tau^{+}\left\{\mathrm{y}^{+}\right\} / \partial \mathrm{y}^{+}$through the viscous layer. The variation of $\tau^{+}\left\{\mathrm{y}^{+}\right\}$in the viscous layer is indicated by evaluating the term $-\mathrm{K}_{\mathrm{py}}{ }^{+}$ at $\mathrm{y}^{+}=30$. In a comparable study, Nieuwstadt and Bradshaw [1997] showed that viscous 
layer statistics can be expected to be approximately equivalent in different geometries if their values of $\operatorname{Re}_{\tau}$ are the same, i.e., $\tau^{+}\left\{\mathrm{y}^{+}\right\}$would be about the same in both geometries.

For fully-developed turbulent pipe flow at high Reynolds numbers, Bejan [1982] derived an "universal" distribution of $\left(\mathrm{S}^{\prime \prime}\left\{\mathrm{y}^{+}\right\}\right)^{+}$by assuming the three-layer von Karman "universal velocity profile." This assumption of an asymptotic high-Reynolds-number profile is equivalent to considering a low streamwise pressure gradient and a constant shear layer $\tau\{\mathrm{y}\}$ near the wall. He predicted that for $\mathrm{y}^{+}>30$ the pointwise entropy generation rate would be less than fifteen per cent of its wall value, decreasing as $\mathrm{y}^{+}$increases (his Figure 3.6), and that the contribution of the turbulent dissipation would be about half the total (his Figure 3.8). As noted by McEligot and Eckelmann [2006], for low values of $-\mathrm{K}_{\mathrm{p}}$ (e.g., "high" Reynolds numbers), the viscous layer of a turbulent channel flow should show the same behavior as the viscous layer in a pipe flow.

\section{Entropy Generation from Turbulent Dissipation}

The objective of the present study is to compare a popular approximate approach estimating S"' for the viscous layer in near asymptotic (high Reynolds number) turbulent flows to the more exact treatment above for the same situation. The DNS database of Kawamura and colleagues for isothermal turbulent channel flow at $\operatorname{Re}_{\tau}=\mathrm{y}^{+} \mathrm{c}=640$ forms the basis [Abe, Kawamura and Matsuo, 2001]; the flow was idealized as being fully-developed between infinitely-wide parallel plates with the Newtonian fluid having constant properties. This condition corresponds to $\mathrm{ReDh} \approx 49,000$ and $\mathrm{K}_{\mathrm{p}} \approx-0.0016$ for the streamwise pressure gradient. According to the order-of-magnitude reasoning of McEligot and Eckelmann [2006], these viscous layer results should be reasonably applicable to any geometry provided the characteristic dimension is greater than about 600 in wall units, $-K_{p}<0.0017$ and $\operatorname{ReDh}>$ 46,000. The reduction in total shear stress across the viscous layer is given by the quantity 30 $\mathrm{K}_{\mathrm{p}}$ or about 4.7 per cent in this case, close to the constant shear layer approximation for an asymptotically-high Reynolds number. The calculations of Kock and Herwig [2004] were for $\operatorname{Re}_{\tau}=395$ giving a reduction of about 7.6 per cent across the viscous layer, i.e., slightly less constant.

The direct numerical simulation solves the governing Navier-Stokes and continuity equations in their three-dimensional, unsteady forms without modeling any terms. Consequently, it is not a "turbulence model." Kawamura and coworkers imposed periodic boundary conditions in the streamwise and spanwise directions with the no-slip condition at the wall. A finite difference method was adopted for solution with a fourth-order central difference scheme for the streamwise and spanwise directions and a second-order central difference scheme in the wall-normal direction. For time advancement, the Crank-Nicholson method was applied for viscous terms with wall-normal derivatives and the second-order Adams-Bashforth method was employed for other terms. The time integration for ensemble averaging corresponded to about fourteen residence times after the flow reached a fullydeveloped state. Spatial resolution for the grid was $\Delta \mathrm{x}^{+}=8.00, \Delta \mathrm{z}^{+}=4.00$ and $\Delta \mathrm{y}^{+}$was varied from 0.15 near the wall to about eight at the centerplane. The staggered computational grid of $1024 \times 256 \times 1024$ nodes covered a volume of $12.8 \mathrm{yc}^{+} \times 2 \mathrm{yc}^{+} \times 6.4 \mathrm{yc}^{+}$. 
From the Background considerations above, one sees that profiles of mean velocity and the dissipation of turbulence kinetic energy are needed in order to calculate the pointwise entropy generation rate. Kawamura and others tabulate a "pseudo dissipation" $\varepsilon_{\mathrm{u}}{ }^{+}$(in the terms of Gersten and Herwig [1992]); citing Hinze [1975], the desired dissipation $\varepsilon^{+}$is called the "true dissipation" by Wilcox [1998]. For a fully-developed channel flow, the difference is provided by a term common with viscous diffusion,

$$
\varepsilon-\varepsilon_{\mathrm{u}}=v\left(\partial^{2} \overline{\mathrm{v}^{2}} / \partial \mathrm{y}^{2}\right)
$$

which, therefore, cancels in the governing equation for turbulence kinetic energy. From the DNS of Kim, Moin and Moser [1987] at $-\mathrm{K}_{\mathrm{p}} \approx 0.006\left(\mathrm{Re}_{\tau} \approx 180\right)$, Bradshaw and Perot [1993] show that the contribution of viscous diffusion is everywhere less than about two per cent of the dissipation rate and conclude that the difference between the true dissipation rate and the pseudo dissipation rate can be ignored for all purposes of computation and discussion.

In the present study we formed $\varepsilon^{+}$by calculating the second derivative of $\left(\overline{v^{2}}\left\{y^{+}\right\}\right)^{+}$ from Kawamura's tabulations and adding it to $\varepsilon_{\mathrm{u}^{+}}$. The term $\left(\overline{\mathrm{v}^{2}}\left\{\mathrm{y}^{+}\right\}\right)^{+}$is positive near the wall and becomes negative near $\mathrm{y}^{+}$about fifteen and positive again at $\mathrm{y}^{+}>160$. The resulting maximum difference between "true" and pseudo dissipation is about 2-1/2 per cent at $\mathrm{y}^{+}$near five -- where it is small relative to direct dissipation from the mean motion anyhow. A maximum negative value of about 1.3 per cent occurs near the edge of the viscous layer.

The direct dissipation from the mean motion (labeled "Mean") and the turbulent dissipation $\varepsilon^{+}$(labeled "Diss") are compared in Figure 1. Figure 1a uses linear coordinates and Figure 1b employs logarithmic coordinates; each has its advantage for the reader. The linear presentation was employed by Bejan for his pipe flow and, perhaps, provides easier visualization of relative magnitudes. The logarithmic representation emphasizes the viscous layer while still giving indication of results well outside it. Logarithmic coordinates also make it easy to estimate per cent differences and, therefore, relative importance of terms.

In wall coordinates the contribution of direct dissipation is unity at the wall. It dominates near the wall while turbulent dissipation contributes about fifteen to twenty per cent to the total. Consequently, the difference between true and pseudo dissipation corresponds to less than one-half per cent of the total in this region. Both direct and turbulent dissipation decrease as $\mathrm{y}^{+}$increases but the reduction of direct dissipation is more rapid with respect to $\mathrm{y}$ than the turbulent dissipation -- so they become of about equal magnitude near $\mathrm{y}^{+}$ $\approx 14$ or so. For larger values of $y^{+}$, turbulent dissipation becomes progressively more dominant. By the edge of the viscous layer the contribution of the mean motion is about negligible. Both direct and turbulent dissipation are seen to decrease rapidly beyond this region. Through the typical logarithmic layer and central core region the dissipation and therefore the entropy generation is essentially provided by the turbulent dissipation. 

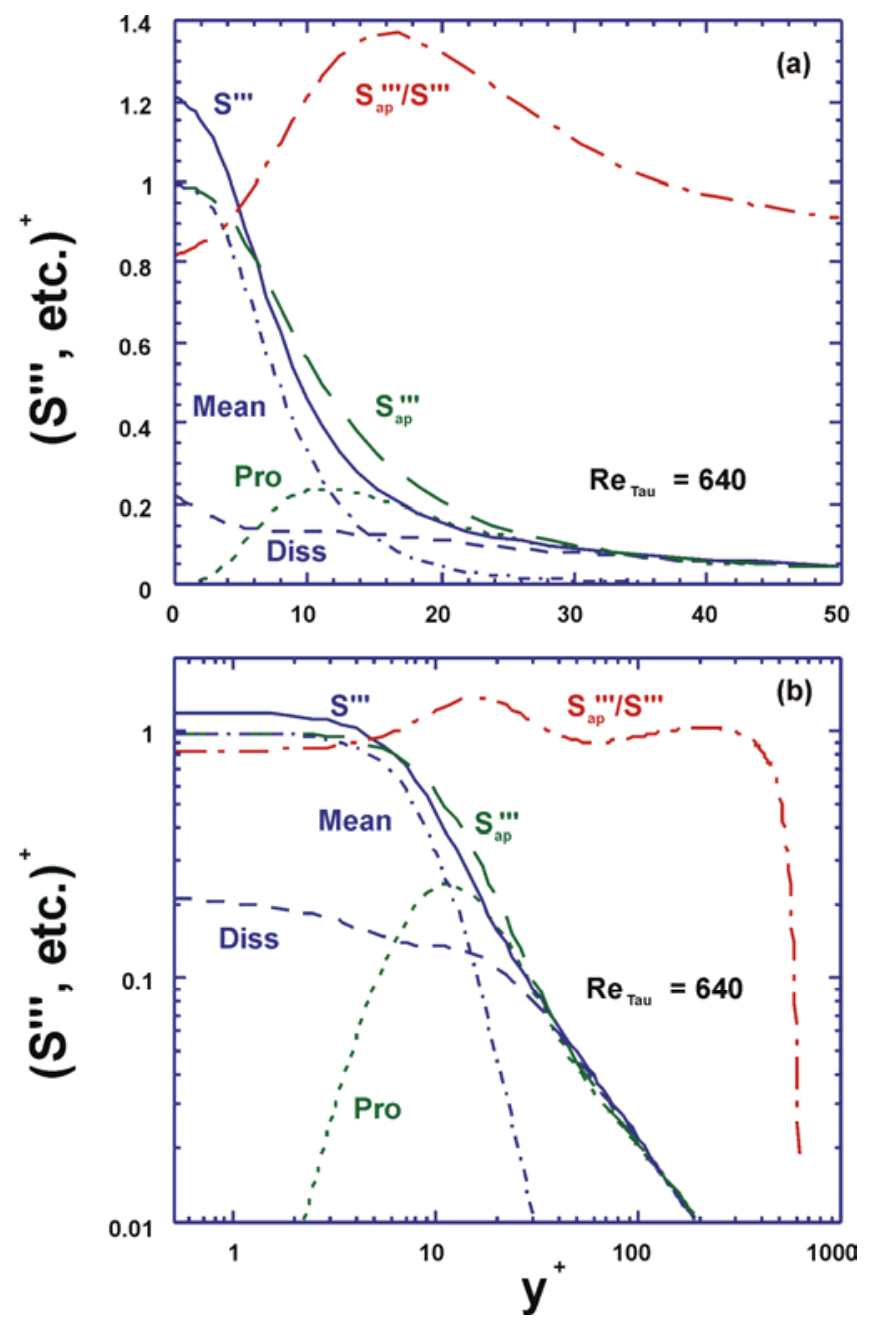

Fig. 1. Predictions of volumetric entropy generation rate and contributing terms from direct numerical simulations of Kawamura and coworkers [Abe, Kawamura and Matsuo, 2001]: (a) linear presentation and (b) logarithmic presentation.

The pointwise entropy generation rate $\left(\mathrm{S}^{\prime \prime}\right)^{+}$is represented by the solid line in Figure 1. Since it is the sum of direct and turbulent dissipation, in wall coordinates it is of order unity in the linear layer $\left(\mathrm{y}^{+}<\sim 5\right)$. It then undergoes a sharp reduction through the rest of the viscous layer. $\mathrm{By}^{+}$of thirty, the volumetric rate $\mathrm{S}^{\prime \prime}$ is reduced to about eight percent of its value at the wall and it continues to decrease rapidly further away from the wall. This result agrees with the approximate prediction of Bejan [1982] for a fully-developed, high-Reynoldsnumber pipe flow; according to the reasoning of McEligot and Eckelmann [2006], his pipe flow would correspond to a low value of $-K_{p}$, the non-dimensional streamwise pressure gradient.

As noted in the Background section, the integral with respect to $y$ of the pointwise entropy generation rate gives the entropy generation rate per unit surface area ( $\left.\mathrm{S}^{\prime \prime}\right)$ which would be sought by thermal fluid engineers. Figure 2 demonstrates the increase of $\left(\mathrm{S}^{\prime \prime}\left\{\mathrm{y}^{+}\right\}\right)^{+}$ through the viscous layer as the solid curve (labeled S"). Since (S"') ${ }^{+}$ranges only from about 1.2 to 0.9 in the linear layer, the integral increases nearly linearly with respect to $\mathrm{y}$ in that 
region. About thirty per cent of the entropy generation occurs in this layer. Beyond $\mathrm{y}^{+} \approx 5$, $\mathrm{S}^{\prime \prime}$ decreases sharply with respect to $\mathrm{y}$. By $\mathrm{y}^{+} \approx 20$ the turbulent dissipation is significantly greater than the direct dissipation due to the mean motion so the contribution to $S$ " is then primarily from turbulent dissipation. For these conditions, the total $\left(\mathrm{S}^{\prime \prime}\left\{\mathrm{y}^{+} \mathrm{c}\right\}\right)^{+}$is about nineteen; by the edge of the viscous layer at $\mathrm{y}^{+} \approx 30$, approximately two-thirds has appeared (and about three-quarters by $\mathrm{y}^{+} \approx 50$ ). Direct dissipation is reduced to less than one per cent of the wall value of total dissipation by $\mathrm{y}^{+} \approx 100$ but by then $\varepsilon^{+}$is an order-of-magnitude greater than the direct dissipation so $\left(\mathrm{S}^{\prime \prime \prime}\right)^{+} \approx \varepsilon^{+}$. Beyond $\mathrm{y}^{+} \approx 100$ the distance to the centerplane is still large in wall units, but $\left(\mathrm{S}^{\prime \prime \prime}\right)^{+}$is small so the additional contribution to entropy generation per unit surface area $\left(\mathrm{S}^{\prime \prime}\right)^{+}$is likewise small.

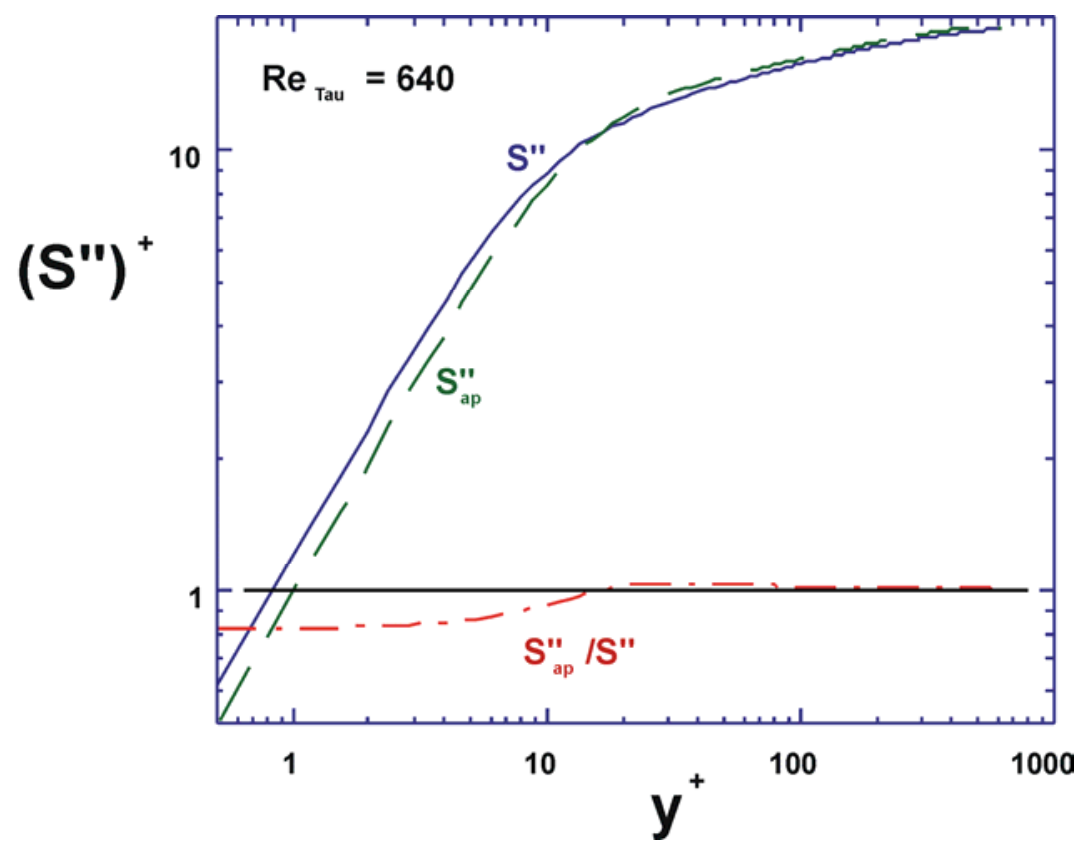

Fig. 2. Predictions of entropy generation rate per unit surface area from direct numerical simulations of Abe, Kawamura and Matsuo [2001].

\section{Approximate Prediction or Measurement of Entropy Generation}

By application of boundary layer and other approximations, Schlichting [eq. 23.8d, 1968 ] has suggested that dissipation in a turbulent boundary layer may be evaluated as

$$
\mu \Phi \approx[\mu(\partial \mathrm{U} / \partial \mathrm{y})-\rho \overline{\mathrm{uv}}](\partial \mathrm{U} / \partial \mathrm{y})
$$

so that the volumetric entropy generation rate can be calculated approximately as

$$
\left(\mathrm{S}_{\mathrm{ap}}{ }^{\prime \prime}\right)^{+} \approx\left(\partial \mathrm{U}^{+} / \partial \mathrm{y}^{+}\right)^{2}+(\overline{\mathrm{uv}})^{+}\left(\partial \mathrm{U}^{+} / \partial \mathrm{y}^{+}\right)
$$

One sees his turbulent dissipation term to be equivalent to the main contributor to production of turbulent kinetic energy. Several investigators have adopted this idea to measure or predict entropy generation (e.g., O'Donnell and Davies [1999], Stieger and Hodson [2003]). While 
the approximation that production is equal to turbulent dissipation may be reasonable in the typical logarithmic layer beyond $\mathrm{y}^{+} \approx 30$, Figure 1 reminds us that they can differ significantly in the viscous layer. Near the wall, production (labeled "Pro") is negligible whereas turbulent dissipation (labeled "Diss") is significant. Then at its peak near $\mathrm{y}^{+}$of eleven, the production is almost eighty per cent greater than turbulent dissipation.

Conceptually, one might suggest that the root source of entropy generation is the production of turbulence kinetic energy which is later dissipated as thermal energy. Further, the approximate representation $\mathrm{S}_{\mathrm{ap}}{ }^{\prime \prime}\{\mathrm{y}\}$ is much easier to predict and measure than the more complete version of Cebeci and Bradshaw [1984], Gersten and Herwig [1992] and others. The Reynolds shear stress (- $\rho \overline{\mathrm{u}} \overline{\mathrm{v}})$ can be measured at a point by single-slanted or dualcrossed hot-wire or hot-film probes and comparable laser Doppler velocimetry systems and, in some situations, it can be deduced from mean velocity profile data (e.g., pitot tubes).

However, for the exact treatment, one needs to measure the instantaneous gradients, i.e., values of the individual components at two points simultaneously. For example, Hinze [1975] suggests three techniques for deducing turbulent dissipation from hot-wire measurements but all depend on isotropic assumptions which are not valid close to a wall. For prediction of $\varepsilon^{+}$ with turbulence models, one needs solution of a dissipation equation (which corresponds to pseudo dissipation $\varepsilon_{\mathrm{u}}$ ) plus solution for the Reynolds normal stress in the wall-normal direction to evaluate the dominant contribution to viscous diffusion. In contrast, even a reasonable van Driest mixing length model can be adequate to predict the Reynolds shear stress in the viscous layer and, therefore, the production of turbulence kinetic energy there [Huffman and Bradshaw, 1972; McEligot, 1985]. So the question becomes --- how well (or poorly) do the two approaches agree?

One sees from the discussion of Figure 1 above that, in the linear layer near the wall, the pointwise volumetric entropy generation rate $S_{a p}{ }^{\prime \prime}\{y\}$ will be less than $S^{\prime \prime \prime}\{y\}$. Then as production exceeds dissipation and direct dissipation becomes the same order-of-magnitude as turbulent dissipation, $\mathrm{S}_{\mathrm{ap}}$ "' $^{\mathrm{y}}\{\mathrm{y}\}$ will become greater than $\mathrm{S}$ "' $\{\mathrm{y}\}$ until they become approximately equal (production $\approx$ dissipation) beyond the viscous layer. At larger distances in the typical logarithmic layer and beyond, both approaches give small values compared to their magnitudes in the viscous layer.

Figure 1 provides comparisons of the two treatments and of the related terms. Figure 1a shows the relation of $S_{a p}$ "' to $S$ "' to be consistent with the reasoning above. It is seen that the distributions in the viscous layer differ between the two approaches as production and dissipation differ considerably there. The approximate technique underpredicts in the "linear" layer and then overpredicts through the rest of the viscous layer. Near the wall the ratio

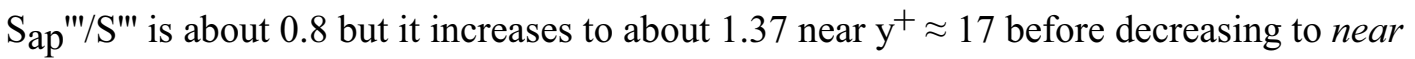
unity in the usual logarithmic region as seen in both figures. Near the "edge" of the viscous layer at $\mathrm{y}^{+}=30$, it is still about ten per cent high. Both approaches agree in the logarithmic layer since production of turbulence kinetic energy approximately equals its dissipation there (in fact, this observation can be employed to derive the logarithmic "law").

Based on a scaling analysis (or boundary layer approximations) for turbulent boundary layers with negligible pressure gradients, Hinze [1975] concludes that in the fully-turbulent region beyond the viscous layer a state of energy equilibrium of the turbulence should occur where production approximately equals turbulent dissipation. Since the contribution to 
entropy generation from the mean motion is the same for $\mathrm{S}_{\mathrm{ap}}$ "' and $\mathrm{S}$ "', this observation by Hinze would imply that $\mathrm{S}_{\mathrm{ap}}{ }^{\prime \prime} \approx \mathrm{S}$ "' in the region beyond the viscous layer. The centerline curve in Figure 1 presents the ratio $\mathrm{S}_{\mathrm{ap}}$ "'/S"' to examine this suggestion; as $\mathrm{y}^{+}$increases, this ratio becomes equivalent to production/dissipation because the contribution from the mean motion becomes negligible. One sees that the two are approximately equal (say within ten per cent) in this region but differ in detail in Kawamura's results. These detailed differences are best seen by examining this ratio over the full range as in Figure 1b.

To the unaided eye, it appears that in Figure 1a the areas under the curves for $\mathrm{S}_{\mathrm{ap}}$ "' and $S^{\prime \prime}$ are approximately equal in the range $0<y^{+}<30$. In the integration to obtain $S^{\prime \prime}\{y\}$, the higher value of $\mathrm{S}^{\prime \prime}$ near the wall counters $\mathrm{S}_{\mathrm{ap}}$ "', which is higher in most of the viscous layer, to give agreement of $\mathrm{S}^{\prime \prime}$ within about four per cent by $\mathrm{y}^{+}=30$. In Figure 2 the curve denoted by centerline symbols provides the ratio $\mathrm{S}_{\mathrm{ap}}$ "/S" to quantify agreement (or disagreement) of $\mathrm{S}_{\mathrm{ap}}$ " with $\mathrm{S}^{\prime}$. Ultimately, the integrations across the channel (e.g., $\mathrm{S}^{\prime \prime}\left\{\mathrm{yc}_{\mathrm{c}}^{+}\right\}$) should be equal. Thus, one sees the asymptote $\mathrm{S}_{\mathrm{ap}} "\left\{\mathrm{yc}_{\mathrm{c}}^{+}\right\}$to be about the same as $\mathrm{S}^{\prime \prime}\left\{\mathrm{yc}_{\mathrm{c}}^{+}\right\}$, consistent with the production of turbulence kinetic energy across the cross section being equal to its turbulent dissipation. At the centerplane, the DNS results predict the difference to be less than one per cent (ideally zero).

These comparisons demonstrate that while the approximate approach can give pointwise errors of $\mathrm{S}_{\mathrm{ap}}$ "' $\{\mathrm{y}\}$ over thirty per cent in the viscous layer, the integrals over the viscous layer agree more closely (i.e., within about four per cent).

\section{Concluding Remarks}

By employing the direct numerical simulations of Kawamura and colleagues, it has been possible to evaluate entropy generation in the viscous layer of turbulent channel flow at a near-asymptotically-high Reynolds number. About two-thirds of the entropy generation occurs in this layer. In the viscous layer an approximate estimate of S"' (based on a suggestion of Schlichting [1968]) does not agree with the result from the more exact evaluation of the turbulent dissipation whereas reasonable agreement is found beyond the viscous layer where mechanical equilibrium (production $\approx$ dissipation) is approached. Highlighting the relative contributions due to viscous dissipation of mean-flow kinetic energy and dissipation of turbulence kinetic energy into thermal energy gives insight into the loss mechanisms. The observation of fair agreement between $S_{a p} "\{30\}$ and S" $\{30\}$ (within about four per cent) may be useful in the development of turbulence models and CFD predictions of entropy generation per unit surface area but such extension is beyond the scope of the present study. The present results may be used, if only indirectly, to compare with entropy generation via heat transfer and to assess alterations in design from an entropy minimization point of view. Since the non-dimensional streamwise pressure gradient $K_{p}$ of the DNS calculated by Kawamura and colleagues can be considered to be low, the results for $\left(\mathrm{S}^{\prime \prime}\left\{\mathrm{y}^{+}\right\}\right)^{+}$and $\left(\mathrm{S}^{\prime \prime}\left\{\mathrm{y}^{+}\right\}\right)^{+}$in the viscous layer should be approximately applicable to turbulent boundary layers with high Reynolds numbers (and negligible pressure gradients) as done by Kock and Herwig [2004] in developing their proposed wall functions. 


\section{Acknowledgments}

We are particularly grateful to Prof. Hiroshi Kawamura of Tokyo University of Science for his insight, guidance and use of his benchmark database on his Internet web site (http://murasun.me.noda.tus.ac.jp/). The study reported was partly supported through Science Foundation Ireland and programs at INL under DoE Idaho Operations Office Contract DEAC07-05ID14517. We thank the Director of IKE at Uni. Stuttgart, Prof. Dr. G. Lohnert, for his kind encouragement and support.

\section{References cited}

Abe, H., H. Kawamura and Y. Matsuo, 2001. Direct numerical simulation of a fully developed turbulent channel flow with respect to the Reynolds number dependence. $J$. Fluids Eng., 123, pp. 382-393.

Bejan, A., 1982. Entropy generation through heat and fluid flow. New York: Wiley.

Bradshaw, P., 1975. An introduction to turbulence and its measurement, 2nd edition.

Oxford: Pergamon.

Bradshaw, P., and J. B. Perot, 1993. A note on turbulent energy dissipation in the viscous wall region. Phys. Fluids A, $\underline{5}$, (12), pp. 3305-6.

Cebeci, T., and P. Bradshaw, 1984. Physical and computational aspects of convective heat transfer. New York: Springer.

Clausius, R., 1887. Die mechanische Wärmetheorie. Braunschweig.

Finnicum, D. S., and T. J. Hanratty, 1988. Effect of favorable pressure gradients on turbulent boundary layers. A.I.Ch.E. Journal, 34, pp. 529-540.

Gersten, K., and H. Herwig, 1992. Strömungsmechanik. Braunschweig: Vieweg.

Guoy, M., 1889. Sur l'energie utilisable. J. Phys., $\underline{8}$, pp. 501-

Hinze, J. O., 1975. Turbulence, 2nd ed. New York: McGraw-Hill.

Huffman, G. D., and P. Bradshaw, 1972. A note on von Karman's constant in low Reynolds number turbulent flows. J. Fluid Mech., 53, pp. 45-60.

Julien, H. L., W. M. Kays and R. J. Moffat, 1969. The turbulent boundary layer on a porous plate: Experimental study of the effects of a favorable pressure gradient. Tech. Rpt. HMT4, Thermosci. Div., Mech. Engr. Dept., Stanford Univ.

Kestin, J. 1980. Availability: the concept and associated terminology. Energy Int. J., $\underline{5}$, pp. 679-

Kim, J., P. Moin and R. D. Moser, 1987. Turbulent statistics in fully developed channel flow at low Reynolds number. J. Fluid Mech., 177, pp. 133-166.

Kline, S. J., W. C. Reynolds, F. A. Schraub and P. W. Rundstadler, 1967. The structure of turbulent boundary layers. J. Fluid Mech., 30, pp. 741-773.

Kock, F., and H. Herwig, 2004. Local entropy production in turbulent shear flows: a highReynolds number model with wall functions. Int. J. Heat Mass Transfer, 47, pp. 22052215.

Kock, F., and H. Herwig, 2005. Entropy production calculation for turbulent shear flows and their implementation in cfd codes. Int. J. Heat Fluid Flow, 26, pp. 672-680.

Lichty, L. C., 1936. Thermodynamics. New York: McGraw-Hill.

London, A. L., 1960. Advanced thermodynamics. Course ME 233, Stanford Univ., Fall.

McEligot, D. M., 1985. Measurement of wall shear stress in favorable pressure gradients. Lec. Notes Physics, 235, pp. 292-303.

McEligot, D. M., and H. Eckelmann, 2006. Laterally converging duct flows. Part 3. Mean turbulence structure in the viscous layer. J. Fluid Mech., 549, pp. 25-59. 
McEligot, D. M., L. W. Ormand and H. C. Perkins, 1966. Internal low Reynolds number turbulent and transitional gas flow with heat transfer. J. Heat Transfer, 88, pp. 239-245. Narasimha, R., and K. R. Sreenivasan, 1979. Relaminarization of fluid flows. Adv. Appl. Mech., 19, pp. 221-309.

Neumann, S. O., J. von Wolfersdorf and B. Weigand, 2005. A numerical study of entropy production rates in a film cooling configuration. GAMM (Gesellschaft für Angewandte Mathematik und Mechanik e.V.)

Nieuwstadt, F. T. M., and P. Bradshaw, 1997. Similarities and differences in turbulent boundary-layer, pipe and channel flows. Boundary-Layer Separation in Aircraft Aerodynamics (Eds.: R. A. W. M. Henkes and P. G. Bakker), Delft: Univ. Press, pp. 15-

O'Donnell, F. K., and M. R. D. Davies, 1999. Measurements of turbine blade aerodynamic entropy generation rate. Proc., I. Mech. E., C557/055, 3rd European Conf. Turbomachinery, London, pp. 43-53.

Onsager, L., 1931. Reciprocal relations in irreversible processes. I. Phys. Rev., 37, pp. 405426.

Patel, V.C., 1965. Calibration of the Preston tube and limitations on its use in pressure gradients. J. Fluid Mech., 23, pp. 185-208.

Prigogine, I., 1978. Time, structure and fluctuations. Science, 201, pp. 777-785.

Reynolds, W. C., and H. C. Perkins, 1970. Engineering thermodynamics. New York: McGraw-Hill.

Schlichting, H., 1968. Boundary layer theory, 6th ed. New York: McGraw-Hill.

Schlichting, H., and K. Gersten, 1997. Grenzschicht-Theorie, 9., völlig neubearbeitete und erweiterte Auflage. Berlin: Springer.

Schlichting, H., and K. Gersten, 2000. Boundary layer theory, 8th revised ed. Berlin: Springer.

Spalart, P. R., 1986. Numerical study of sink-flow boundary layers. J. Fluid Mech., 172, pp. 307-328.

Stieger, R. D., and H. P. Hodson, 2003. Unsteady dissipation measurements on a flat plate subject to wake passing. Presented at 5th Euro. Turbomachinery Conf., Prague.

Stodola, A., 1910. Steam and gas turbines. New York: McGraw-Hill.

Wilcox, D. C., 1998. Turbulence modeling for CFD, 2nd ed. La Canada, Cal.: DCW Industries. 